\title{
Identification and study of systems of galaxies in the Shapley supercluster ${ }^{\star}$
}

\author{
C. J. Ragone ${ }^{1,2}$, H. Muriel ${ }^{1,2}$, D. Proust ${ }^{3}$, A. Reisenegger ${ }^{4}$, and H. Quintana ${ }^{4}$ \\ ${ }^{1}$ Grupo de Investigaciones en Astronomía Teórica y Experimental, IATE, Observatorio Astronómico, Laprida 854, Córdoba, Argentina \\ 2 Consejo de Investigaciones Científicas y Técnicas de la República Argentina \\ e-mail: [cin; hernan]@oac .uncor .edu \\ 3 GEPI, Observatoire de Paris-Meudon, 92195 Meudon Cedex, France \\ e-mail: Dominique.Proust@obspm.fr \\ 4 Departamento de Astronomía y Astrofísica, Pontificia Universidad Católica de Chile, Casilla 306, Santiago 22, Chile \\ e-mail: [areisene; hquintana] @astro.puc.cl
}

Received 13 June 2005 / Accepted 7 September 2005

\section{ABSTRACT}

Based on the largest compilation of galaxies with redshift in the region of the Shapley Supercluster (Proust et al. 2004, P2004), we identified 122 galaxy systems, 60 of which are new systems. Using the SuperCOSMOS catalogue, we have assigned $b_{j}$ magnitudes to each galaxy in our compilation. The sample of galaxy systems was used to estimate the mass function of systems in the range $10^{13}$ to $10^{15} M_{\odot} h^{-1}$. We computed a lower value to the total mass in the region of the Shapley Supercluster with this mass function. Using 15 mock catalogues we derived the mean mass that these kinds of systems have before comparing it with that obtained from the real data.

Key words. galaxies: clusters: general

\section{Introduction}

The Shapley Supercluster (hereafter SSC) has the highest concentration of clusters and groups of galaxies in the local universe. It is centrally dominated by three Abell clusters (A3556, A3558, and A3562) and contains more than 20 Abell clusters. This supercluster has an extent of $\sim 20 \mathrm{Mpc}$, or even larger, and has not yet virialized. Kull \& Böhringer (1999) analyzed a region of $6 \times 3$ degrees and found evidence of X-ray emission connecting the three central clusters of the SSC, indicating that the inter-cluster gas is being warmed up. Therefore, the core of the supercluster is in the procces of virialization.

A mass estimate of the SSC is particularly interesting due to its proximity to the local group. Moreover, it can be used to constrain theoretical models that should be able to predict this type of mass concentrations. Nevertheless, the fact that superclusters are not virialized systems seriously limitats the possible methods that can be used to estimate the mass. Bardelli et al. (2000) used galaxy counts to suggest that the SSC has a significantly higher mean density than the cosmic average. Reisenegger et al. (2000) (R2000 hereafter) applied the spherical collapse model and found that the mass enclosed by a radius of $8 h^{-1} \mathrm{Mpc}$ is in the range $2.0 \times 10^{15}$ to $1.3 \times 10^{16} M_{\odot} h^{-1}$,

* Tables 1 and 2 are only available in electronic form at http://www.edpsciences.org representing an overdensity range $\rho / \rho_{\mathrm{c}} \sim 3-20$. In this work we explore an alternative way to estimate a lower limit to the total mass of the SSC. In a high mass concentration like the SSC, a significant fraction of galaxies are supposed to belong to groups or clusters of galaxies and, therefore, the mass function of these systems can be used to provide a lower limit to the total mass of the supercluster. In order to select a sample of galaxy systems that is as complete as possible, we used the resent catalogue by Proust et al. (2004, hereafter P2004). These authors compiled the largest velocity catalogue in the region of the SSC. It contains radial velocities for 8341 galaxies and represents a powerful tool for identifying and studying the galaxy systems in the SSC.

Large redshift surveys like 2dFGRS (2 degree Field Galaxy Redshift Survey) and SDSS (Sloan Digital Sky Survey) have stimulated the identification of galaxy groups based on the friends-of-friends algorithm (FOFA). The original algorithm introduced by Huchra \& Geller (1982) was modified by Merchán \& Zandivarez (2002) to take the sky coverage of 2dFGRS into account. Using mock catalogues, this technique has been extensively tested and has demonstrated its robustness in identifying galaxy groups in redshift space (see for instance Nolthenius \& White 1987).

The aim of this work was to identify and study galaxy systems in a $12 \times 15$ degrees region of the SSC, based on the 
redshift compilation of $\mathrm{P} 2004$. The resulting catalogue was used to estimate a lower boundary to the total mass of the SSC. The outline of this paper is as follows. A description of the SSC is given in Sect. 2. Details of the simulation and the mock catalogue construction are described in Sect. 3. A brief description of the identification process for both observational data and mock catalogues is presented in Sect. 4. In Sect. 5 we analyse and estimate the mass enclosed by the SSC, and finally in Sect. 6 we discuss our main conclusions.

\section{Observational data}

The compilation of P2004 contains 8341 galaxies with redshifts taken from Quintana et al. (1995, 1997, 2000), Proust et al. (2004), Drinkwater et al. (1999, 2004), Watson et al. (2000), Bardelli et al. (1998, 2000, 2001), Kaldare et al. (2003), and data from the NED NASA/IPAC database. The P2004 catalogue covers a region of $12 \times 30$ degrees on the sky, where half of the galaxies are members of the SSC, with velocities within 9000 and $18000 \mathrm{~km} \mathrm{~s}^{-1} \mathrm{~s}^{-1}$. The main region is centered at $v=14500 \mathrm{~km} \mathrm{~s}^{-1}$.

After taking the numerous sources involved in the P2004 compilation into account, a non-uniform redshift completeness is expected. The P2004 compilation overlaps the SuperCOSMOS Sky Survey (hereafter SSS) completely. Therefore, in order to test the redshift incompleteness we have assigned the corresponding magnitude in the SSS survey to each galaxy in the P2004 catalogue. Since none of the sources of the P2004 compilation includes faint galaxies, the cross correlation with the SSS was performed selecting galaxies brighter than $b_{j}=19$. Using a matching radius of 10 arcsec, we found a positive cross-correlation between P2004 and SSS for more than $96 \%$ of the galaxies. Of these objects, $14 \%$ correspond to multiple identifications. After visually inspecting several images of multiple identifications we introduce several criteria based on the matching radius and the magnitudes in order to correctly assign the SSS galaxy. We estimated that more than $95 \%$ of the Shapley galaxies have been correctly identified in the SSS.

With the aim of performing a completeness mask, we used the HEALPix tool to produce an equal-area pixelisation of the sky. For each pixel of $2.6 \times 10^{-4}$ strd, we counted galaxies up to a designed apparent magnitude limit, defining the completeness in a pixel as the ratio of the number of galaxies in the SSC to the number of galaxies in the SSS survey. The resulting mean completeness for an apparent magnitude limit of 17.5 is $\sim 0.5$. The fraction of galaxies with measured redshift significantly decreases for fainter magnitudes. It should be noted that the completeness is not uniform, so it shows a tendency to be higher in the high density regions of the SSS.

\section{Mock catalogues}

In this study we used one of the publicly available Very Large Simulations (VLS) (Yoshida et al. 2001). Briefly, this simulation models a box of $479 \mathrm{Mpc} h^{-1}$ in size, with $512^{3}$ particles, each of mass $6.86 \times 10^{10} M_{\odot} h^{-1}$. Given the success of the model at reproducing observational data, we chose the $\Lambda C D M$ scenario with cosmological parameters: $\Omega_{\mathrm{m}}=0.3, \Omega_{\Lambda}=0.7$, normalization $\sigma_{8}=0.9$, and $H_{0}=70 \mathrm{~km} \mathrm{~s}^{-1} \mathrm{Mpc}^{-1}$.

Due to the large box size, it was possible to find a large number of rich systems, so we chose those that represent high density regions. R2000, using estimates of the SSC mass enclosed by $8 \mathrm{Mpc} h^{-1}$, find a density range $\rho / \rho_{\mathrm{c}} \sim 3-20$. The lower limit of this range corresponds to the Diaferio and Geller method (1997), whilst the upper one is obtained using the spherical collapse model (for more details see R2000).

Following these authors, we searched in the simulation for overdense regions in spheres of a radius of $8 \mathrm{Mpc}^{-1}$ and selected 14 of them with overdensities in the mentioned range. We refer positions to a coordinate system situated $145 \mathrm{Mpc} h^{-1}$ away from the sphere center in order to mimic the real position of the SSC, and we selected a cone-shaped volume which subtended $20 \mathrm{Mpc} h^{-1}$ of radius at the distance of the sphere center. Within this volume, overdensities ranged from $\rho / \rho_{\mathrm{c}} \sim 0.5$ to 1.1. Finally, we assigned particles within this volume a luminosity function that can be described by a Schechter fit with parameters $\alpha=-1.19$ and $M_{\mathrm{b}}^{*}=-19.79$ (Madgwick et al. 2002). The adopted $k+e$ correction was taken from (Norberg et al. 2001) and is given by the equation

$k(z)+e(z)=\frac{z+6 z^{2}}{1+20 z^{3}}$

A set of 15 mock catalogues was constructed from this data. Galaxies were selected within the described volume by applying the apparent magnitude limit of the SSC survey. The redshift incompleteness of the SSC was not reproduced in the mock catalogues due to the non-uniformity of the sample (i.e. high density regions show a higher completeness on average). In the following analysis, we select galaxies brighter than $m_{\mathrm{b}}=17.5$ in both mocks and real data. The mean redshift completeness for the SSC subsample is $\sim 0.5$.

\section{Identification of galaxy groups}

The completeness mask described in Sect. 2 allows us to identify galaxy groups in the SSC following the procedure described in Merchán \& Zandivarez (2002). According to these authors, the identification can be performed using a similar algorithm similar to the one developed by Huchra \& Geller (1982) but modified in order to take the incompleteness of the galaxy sample into account by introducing a correction in the linking lengths' scale factor. The algorithm links pairs of galaxies according to the following conditions:

$D_{12}=2 \sin \left(\frac{\theta_{12}}{2}\right) \frac{V}{H_{0}} \leq D_{L}=D_{0} R$

$V_{12}=\left|V_{1}-V_{2}\right| \leq V_{L}=V_{0} R$

where $\theta_{12}$ and $V$ are the angular separation and the mean radial velocity, respectively; $D_{0}$ and $V_{0}$ are the transversal and radial linking cut-off at the fiducial velocity $V_{\mathrm{f}}$. Scale factor $R$ is introduced to compensate for the decline in the selection 
function with distance. In this case the scaling factor is computed as

$R=\left[\frac{\int_{\infty}^{M_{12}} \phi(M) \mathrm{d} M}{\int_{\infty}^{M_{\lim }} \phi(M) \mathrm{d} M} \frac{\left(C_{1}+C_{2}\right)}{2}\right]^{-\frac{1}{3}}$

where $M_{\text {lim }}$ and $M_{12}$ are the absolute magnitude of the brightest galaxy visible at distances $V_{\mathrm{f}} / H_{0}$ and $V / H_{0}$, respectively. The $\left(C_{1}+C_{2}\right) / 2$ factor in the previous equation corresponds to the correction introduced to the original Huchra \& Geller (1982) algorithm to take the incompleteness of the sample into account, $C_{1}$ and $C_{2}$ are the completeness values in the direction of each galaxy. Finally $D_{0}$ and $V_{0}$ are the corresponding linking lengths at the fiducial velocity $V_{\mathrm{f}}$ and $\phi(M)$ is the luminosity function of the sample.

In this particular case, given the irregularity of the sky coverage, the remarkably rich concentration of galaxies and the large mass system range in the SSC, we need to take special care override misidentifications and spurious merging. In order to optimize the identification of systems, we took into account the X-ray flux contour plot of the Shapley Supercluster core by Kull \& Böhringer (1999). We performed a series of system identifications in this same region using different linking length values $V_{0}$ and $D_{0}$, where $D_{0}$ is related to the number density contour surrounding a group relative to the mean number density $(\delta \rho / \rho)$.

We found that standard values of $\delta \rho / \rho(\sim 80)$ caused the two central groups (SC1329-313 and SC1327-312) in the Kull \& Böhringer (1999) plot to merge into a single system. In order to find a fair number of groups and to ensure that most of them are single dynamical systems, we selected the lowest overdensity that does not merge those X-ray systems that appear as single systems. Despite this, this choice yields a high value of $\delta \rho / \rho \sim 400$, which ensures small groups will be identified in a correctly.

Figure 1 shows the best correlation between the identified systems and the X-ray contours of Kull \& Böhringer (1999). Identification of these systems corresponds to a transversal and a line-of-sight linking lengths of $D_{0}=0.14 \mathrm{Mpc} h^{-1}$ and $V_{0}=$ $200 \mathrm{~km} \mathrm{~s}^{-1}$, respectively.

As a result of the identification process we found 122 systems of galaxies containing at least 4 members. The number of systems drops to 73 when considering groups within the $9000<v r<18000$ range. The spatial distribution of these groups is illustrated in Fig. 2, whereas Fig. 3 shows the distribution normalized to the total number of groups of their main properties: radial velocity, velocity dispersion, virial radius, and virial mass. In the same figure we plot (dotted line) the distributions corresponding to groups identified in the mock catalogues, again normalized to the total number of groups in the mocks. We can see a lack of low-mass systems in the SSC distributions, which is likely to be produced by the bias in the completeness of the sample. In Fig. 4, groups (dots) are overlapped with the completeness mask, Where we can appreciate a tendency of groups to correlate with the regions of higher completeness. This effect is the result of at least two processes: i) the FOFA algorithm tends to be more efficient in the regions of higher completeness and; ii) the compilation of P2004 has higher completeness in high density regions, where most of the galaxy systems are present.

The following are median values of the properties listed before for both mock and real samples, median dynamical properties of SSC groups are higher given the lack of small systems mentioned above:

$\begin{array}{ll}V r_{\mathrm{SSC}}=14597.8 \mathrm{~km} \mathrm{~s}^{-1} & V r_{\text {mock }}=14370.9 \mathrm{~km} \mathrm{~s}^{-1} \\ \sigma_{\mathrm{SSC}}=344.6 \mathrm{~km} \mathrm{~s}^{-1} & \sigma_{\text {mock }}=200.8 \mathrm{~km} \mathrm{~s}^{-1} \\ R_{\mathrm{vir}-\mathrm{SSC}}=0.78 \mathrm{Mpc}^{-1} & R_{\mathrm{vir}-\text { mock }}=0.57 \mathrm{Mpc}^{-1} \\ M_{\mathrm{vir}-\mathrm{SSC}}=4.6 \times 10^{13} M_{\odot} h^{-1} & M_{\text {vir-mock }}=1.4 \times 10^{13} M_{\odot} h^{-1} .\end{array}$

Table 1 shows the different properties of the galaxy systems identified in the SSC sample. As noted before, if we restrict the sample to the Shapley Supercluster region $(9000<$ $\left.v_{\mathrm{r}}<18000\right)$, the resulting number of systems is 73 . We performed a cross correlation between our catalogue and the NED NASA/IPAC database, using a search radius of 20 arcsec and a $\Delta v_{\mathrm{r}} \leq 2000 \mathrm{~km} \mathrm{~s}^{-1}$. The last restriction was only applied to those systems in the NED with known redshift. We found that 60 of the 122 are new galaxy systems. Of the 62 already known systems, 37 were identified as clusters, althoug, many of them were also identified as group. (34 are Abell clusters). The remaining 25 were only identified as groups. Table 2 shows the name of the systems (Col. 2), the type of system, the mean radial velocity, and the difference in the central position (Cols. 3-5, respectively).

\section{Mass statistics}

In this section we compute the mass function of groups in the SSC and use it to estimate a lower limit to the total mass of this system. We used the mock catalogues and the simulation described in Sect. 3 in order to compute the real mass enclosed in a volume like the SSC one and to compare with the inferred mass. The group mass function was derived using the $1 / V \max$ method, which has the advantage of being a non-parametric procedure, i.e. it does not assume a shape of the mass function. Moreover, it not only gives shape but also normalization at the same time. The differential mass function can be computed using the equation

$\frac{\mathrm{d} N}{\mathrm{~d} \mathcal{M}}(\mathcal{M})=\sum_{\left|\mathcal{M}_{i}-\mathcal{M}\right| \leq d \mathcal{M} / 2} \frac{1}{V_{\max }\left(M_{i}\right)}$

where $\mathcal{M}_{i}$ and $M_{i}$ are the group mass and absolute magnitude respectively, and

$V_{\max }\left(M_{i}\right)=\int_{\min \left(z_{2}, z_{\max }\right)}^{\max \left(z_{1}, z_{\min }\right)} \frac{\mathrm{d} V}{\mathrm{~d} z} \mathrm{~d} z$

is the maximum comoving volume, within the catalogue velocity cut-off, out to which the group $i$ with magnitude $M_{i}$ remains observed. Both $z_{1}$ and $z_{2}$ are the geometric limits of the sample, and $z_{\min }$ and $z_{\max }$ are found by solving the equations

$M_{i}=\left\{\begin{array}{l}m_{2}-5 \log d_{L}\left(z_{\max }\right)-25-k\left(z_{\max }\right) \\ m_{1}-5 \log d_{L}\left(z_{\min }\right)-25-k\left(z_{\min }\right)\end{array}\right.$

hence, $z_{\min }$ and $z_{\max }$ are the redshift of groups whose absolute magnitudes are identical to the one of the object being considered, but its apparent magnitudes are the bright $\left(m_{1}\right)$ and faint $\left(m_{2}\right)$ limits of the survey. 


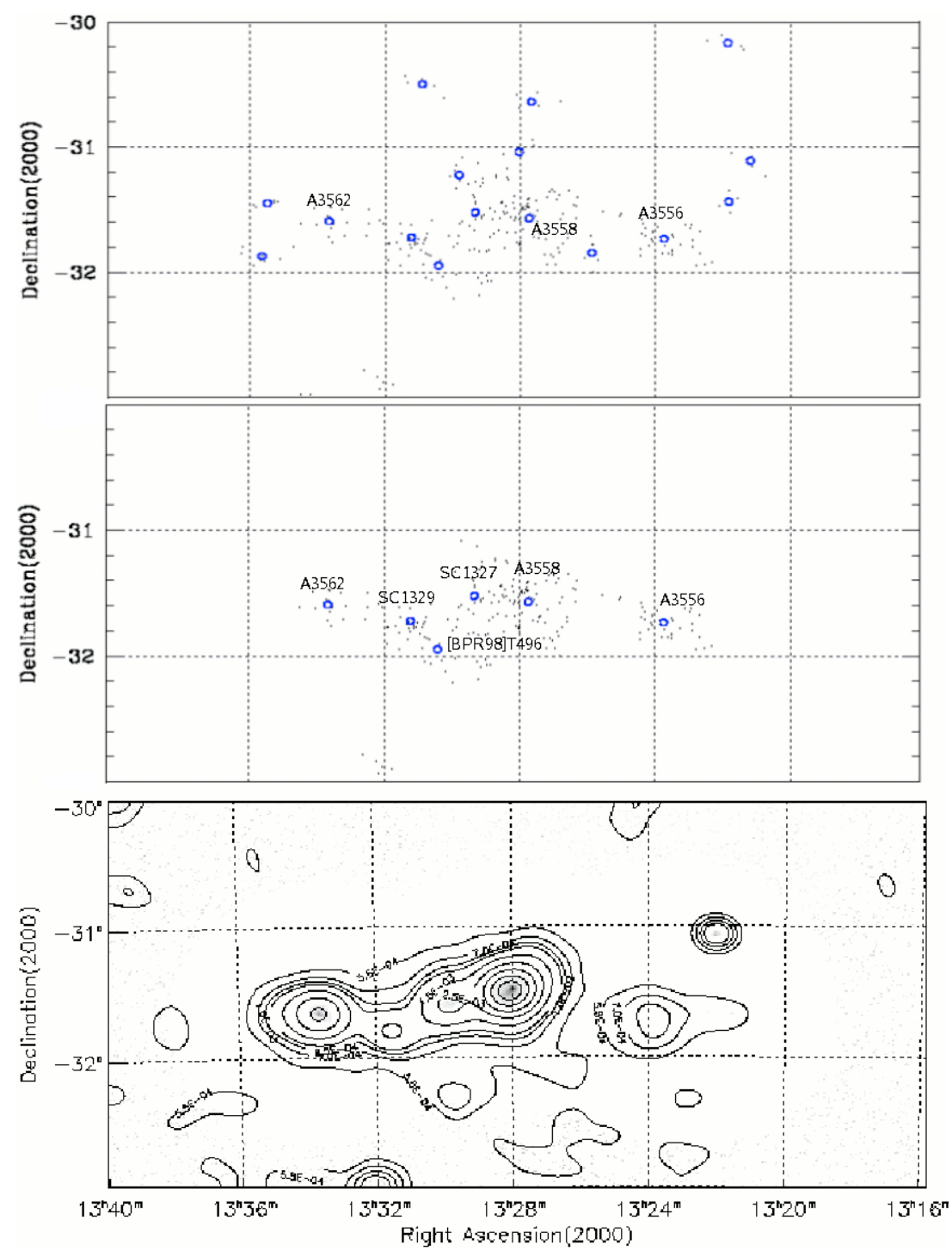

Fig. 1. Lower panel: X-ray contours of Kull \& Böhringer (1999), for the central region of the SSC. Middle panel: SSC groups with at least 10 galaxies in the same region. Top panel: the same as the middle panel but considering groups with at least four members. Dots represent members of galaxy group and open circles the group centers.

Before using the $1 / V_{\max }$ method, there are three intermediate issues to resolve: namely, to calculate groups "apparent magnitudes", which can be inferred from groups luminosities, to find out the corresponding completeness limit of the sample, and to determine the geometric limits where the mass function is to be calculated. Regarding the former, we calculated the total luminosity $L_{\text {tot }}$ for each group using the prescription outlined in Moore et al. (1993), as the sum of the luminosities of its galaxy members $\left(L_{\mathrm{obs}}\right)$ plus the integrated luminosity of galaxies below the magnitude limit of the sample $\left(L_{\text {cor }}\right)$ :

$L_{\mathrm{tot}}=L_{\mathrm{obs}}+L_{\mathrm{cor}}$.

$L_{\text {corr }}$ is calculated assuming that group members are independently drawn from a Schechter fit to the luminosity function of galaxies in groups. Hence, for faint group members which can not be observed

$L_{\text {cor }}=N_{\text {obs }} \frac{\int_{0}^{L_{\text {lim }}} L \Phi(L) \mathrm{d} L}{\int_{L_{\mathrm{lim}}}^{\infty} \Phi(L) \mathrm{d} L}$ where $L_{\lim }=10^{0.4\left(M_{\odot}-M_{\lim }\right)}, M_{\lim }=m_{\lim }-25-5 \log \left[d_{L}(z)\right]$, and $M_{\odot}$ is the absolute magnitude of the sun in the corresponding band. Once the total luminosity of a group is obtained, we were able to compute its absolute magnitude, and finally, the group "apparent magnitude" using the distance modulus. As it relates to the "apparent magnitude" limit of the group sample, we used the completeness test developed by Rauzy (2001), which can be applied to any redshift-space distribution and used for any selection function. It is assumed that the luminosity function of the population depends neither on time nor on the spatial position of the objects; peculiar velocities of the observed redshifts are negligible, and the sample is complete in apparent magnitude up to a given magnitude limit $m_{\mathrm{lim}}$.

The principle of the method consists of defining the random variable

$\zeta=\frac{F(M)}{F\left[M_{\mathrm{lim}}(Z)\right]}$ 

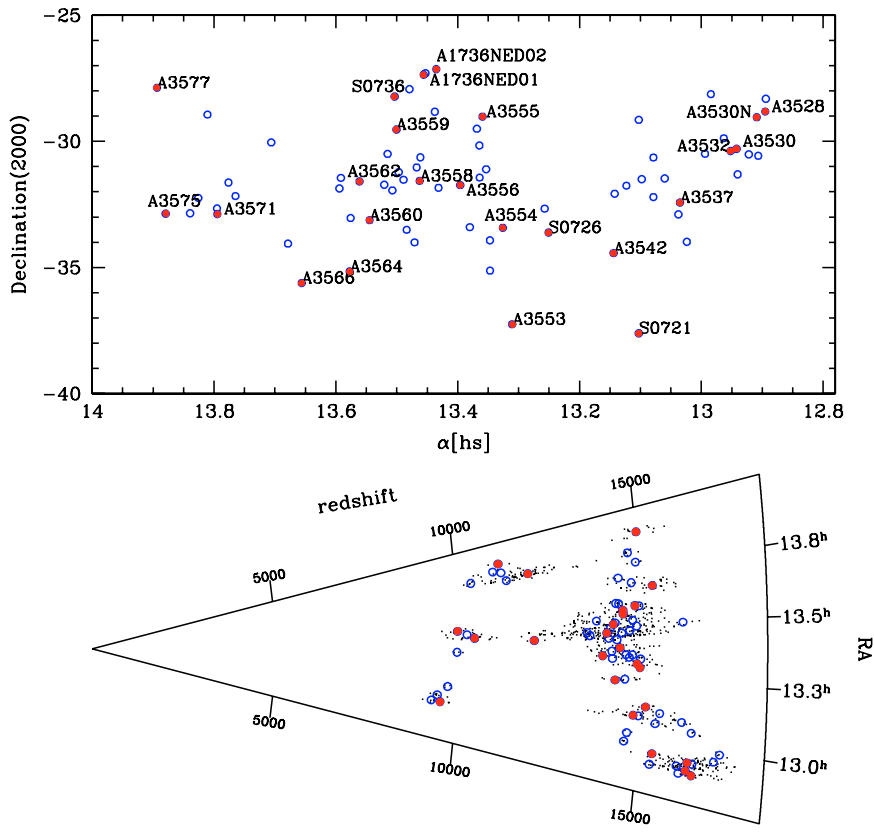

Fig. 2. The spatial distribution of groups with at least 4 members and within a velocity range $9000<V_{\mathrm{r}}<18000$. Open circles represent group centers and filled circles the known ACO clusters; in the lower panel dots correspond to members of galaxy group.
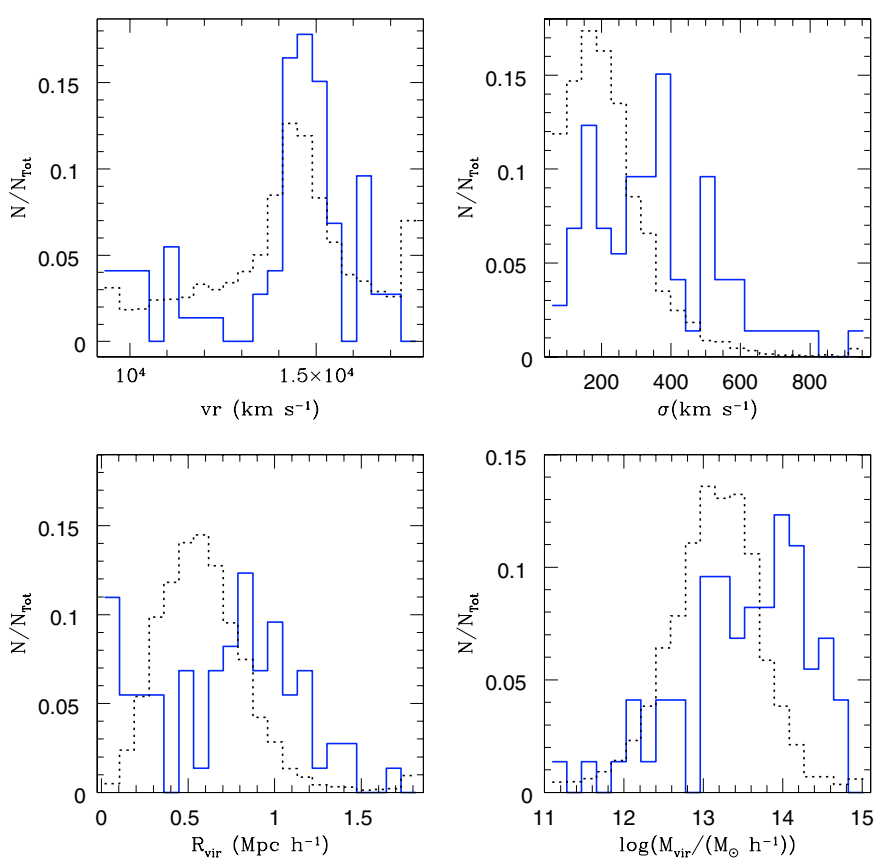

Fig. 3. Histograms showing the distributions of groups redshifts (upper left panel), velocity dispersions (upper right panel), virial radii (lower left panel), and virial masses (lower right panel). Only groups with at least 4 members and within the velocity range $9000<V_{\mathrm{r}}<$ 18000 were included. In all cases solid and dashed lines correspond to SSC and mock groups, respectively.

where $F(M)$ stands for the cumulative luminosity function, and $M_{\lim }(Z)=m_{\lim }-Z$, where $Z$ is the distance modulus. The

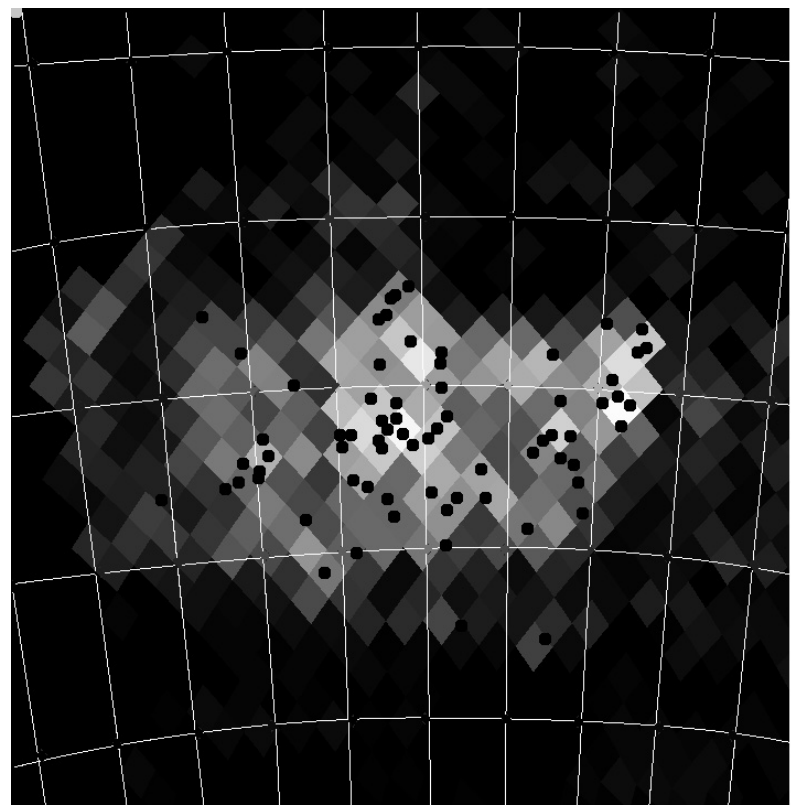

$-20.0$

$-25.0$

$\begin{array}{llllllllll}14.4 & 14.2 & 14.0 & 13.8 & 13.4 & 13.2 & 13.0 & 12.8 & 12.6 & 12.4\end{array}$

\section{$-30.0$}

$-35.0$

$-40.0$

Fig. 4. Identified groups containing at least 4 members within the velocity range $9000<V_{\mathrm{r}}<18000$, overlapped with the completeness mask derived in Sect. 2. Both $\alpha$ and $\delta$ units are hours and degrees, respectively. The bottom gray scale bar corresponds to the completeness level.

variable $\zeta$ can be estimated without any prior knowledge of $F(M)$, considering for each object the quantity

$\hat{\zeta}=\frac{r_{i}}{n_{i}+1}$

where $r_{i}$ is the number of objects satisfying $M \leq M_{i}$ and $Z \leq$ $Z_{i}$, and $n_{i}$ is the number of objects such that $M \leq M_{\lim }^{i}\left(Z_{i}\right)$ and $Z \leq Z_{i}$. The expectation of $\hat{\zeta}$ is $E_{i}=1 / 2$ and its variance $V_{i}=\frac{n_{i}-1}{12\left(n_{i}+1\right)}$. Hence, the quantity

$T_{\mathrm{C}}=\frac{\sum_{i=1}^{N_{\mathrm{gal}}}\left(\hat{\zeta}_{i}-\frac{1}{2}\right)}{\left(\sum_{i=1}^{N_{\text {gal }}} V_{i}\right)^{\frac{1}{2}}}$

has an expectation of zero and variance unity. The test requires the computation of $T_{\mathrm{C}}$ for subsamples that are truncated up to increasing values of the limiting apparent magnitude $m_{*}$. The $T_{\mathrm{C}}$ statistic is expected to be distributed around zero with dispersion of unity, as long as $m_{*}$ remains below the completeness limit $m_{\mathrm{lim}}$. If $m_{*}$ becomes greater than $m_{\mathrm{lim}}$, there will be a lack of objects with $M$ fainter than $M_{\text {lim }}$, resulting in a systematic decline of $T_{\mathrm{C}}$ towards negative values.

Figure 5 shows the result of aplying the $T_{\mathrm{C}}$ statistics to the SSC (solid line) and mock groups (dashed line). A systematic decline of the $T_{\mathrm{C}}$ statistics can be observed beyond $m=14.3$ for SSC (vertical solid line). For groups in the M1 sample, the apparent magnitude cut-off is $m=14.5$ (vertical dashed line). Horizontal lines are the $1 \sigma$ limits of the $T_{\mathrm{C}}$ variance.

Finally, regarding the geometric limits of our fluxlimited sample, we adopted the velocity range defined by 


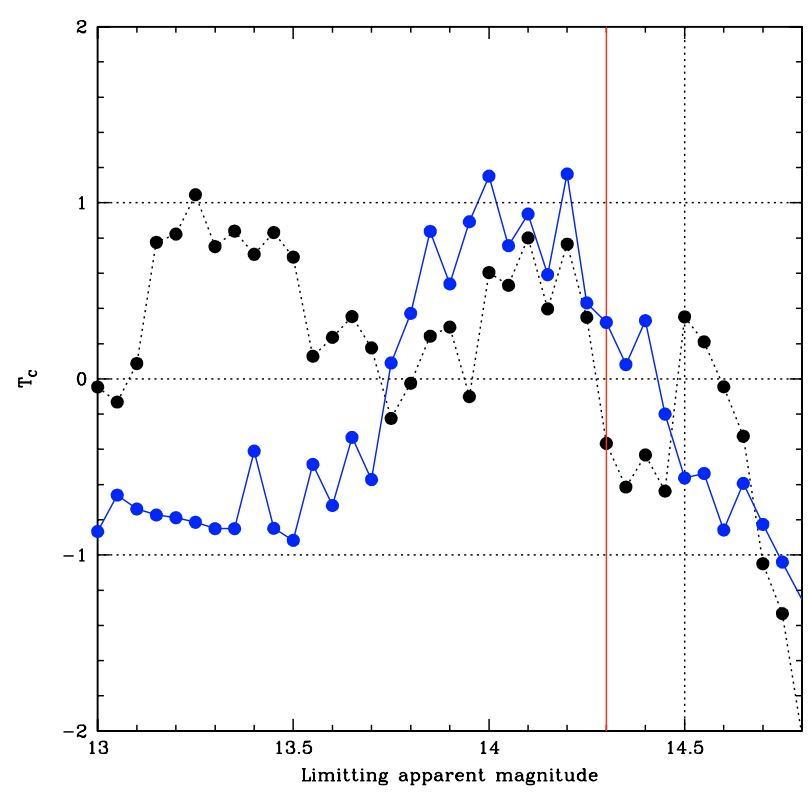

Fig. 5. Completeness test of Rauzy (2001). Solid and dotted lines correspond to SSC and mock groups, respectively. The vertical solid line denotes the adopted apparent magnitude limit for SSC, whereas the dotted vertical line is associated to mock groups. Horizontal lines are the $1 \sigma$ limits of the $T_{\mathrm{C}}$ variance.

Quintana et al. (2000), who consider as SSC members all galaxies in the redshift range $9000<v<18000$. Considering both, these redshift limits and the "apparent magnitude" cutoff, we computed the SSC mass function with 55 galaxy groups containing at least 4 members.

Results are shown in Fig. 6, where the SSC group mass function (solid line) is compared to the one obtained from the mock groups (dotted line, 2729 groups selected from a complete flux-limited sample of galaxies). We found that the obtained mass functions have different trends up to $M \sim 1 \times$ $10^{14} M_{\odot} h^{-1}$, while they are quite similar in the high mass region. The lack of groups in the low mass region of the SSC mass function was expected considering that the completeness is higher close to massive clusters and lower in the underdense areas of the SSC. Consequently, low mass groups are likely to be lost by the group finding process. Analysis of Fig. 6 leads us to conclude that the completeness mask is the primary responsible for the differences between the SSC and mock mass functions. Following this idea, and assuming that our mock catalogues correctly represent the SSC and then integrating the mock mass function in the considered volume, all should provide an estimate of the mass that is typical of systems like the SSC. The same procedure applied to the SSC group mass function yields a minimum mass, since as noted some of the SSC low mass groups are possibly missed given they have been poorly sampled. After performing these computations we obtained a mass of $\sim 1.1 \times 10^{16} M_{\odot} h^{-1}$ from the mock mass function, whereas the mass drops to a value of $4.8 \times 10^{15} M_{\odot} h^{-1}$ for the SSC; both of these masses consider systems with at least $1 \times 10^{13} M_{\odot} h^{-1}$.

Using the high density regions selected in the simulations, we find that $\sim 60 \%$ of the particles in a $8 \mathrm{Mpc}^{-1}$ sphere are

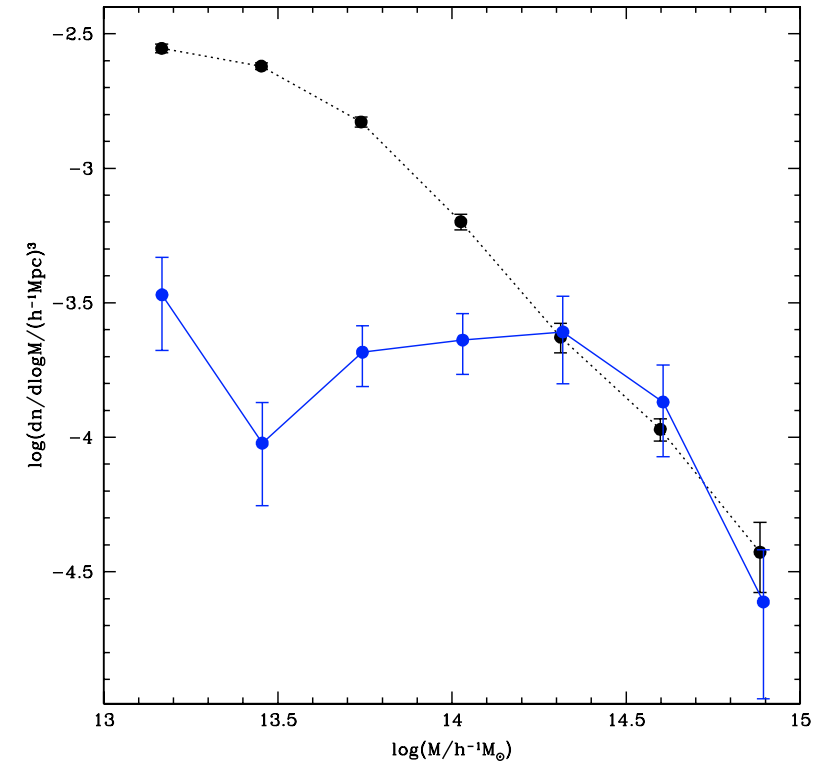

Fig. 6. The mass function for SSC (solid line) and mock groups (dotted line). Error bars were estimated by the bootstrap resampling technique.

within groups of at least $1 \times 10^{13} M_{\odot} h^{-1}$, whereas this value drops to $\sim 30 \%$ when considering the cone volume.

We can give a rough estimation of the total mass enclosed in the cone-volume we are using by considering the fraction of mass that, according to the simulation, we are missing. The final values that we find for our mock catalogues and the SSC are $3.7 \times 10^{16}$ and $1.6 \times 10^{16}$, respectively.

R2000, using a spherical collapse model, state that the SSC mass enclosed by a radius of $8 \mathrm{Mpc} h^{-1}$ lies between $2 \times$ $10^{15}$ and $1.3 \times 10^{16} M_{\odot} h^{-1}$. Disregarding this for groups peculiar motions, we can roghly compute the mass within a sphere of radius $8 \mathrm{Mpc} h^{-1}$, centered as in R2000, by summing up the group virial masses. In so doing we obtain $2.3 \times 10^{15} M_{\odot} h^{-1}$, If we take into account the $40 \%$ of the mass that is not in galaxy systems, our derived value is $3.8 \times 10^{15}$ for the SSC in a $8 \mathrm{Mpc} h^{-1}$ sphere, a result in fair agreement with R2000.

\section{Conclusions}

Based on the compilation of redshift made by Proust et al. (2004), and assigning magnitudes from the SuperCOSMOS catalogue, we have identified galaxy systems in an area of $12 \times 15$ degrees. The transversal and line-of-sight linking lengths used to identified systems were $D_{0}=0.14 \mathrm{Mpc} h^{-1}$ and $V_{0}=200 \mathrm{~km} \mathrm{~s}^{-1}$, respectively. We identified 122 systems of galaxies with at least 4 members, and 73 of these are in the Shapley redshift range $\left(9000<v_{\mathrm{r}}<18000\right)$. 62 of the 122 are new systems. Of the remaining, 37 were previously identified as clusters (34 are Abell clusters). The velocity dispersion for the whole sample of galaxy systems spans a range from $\sim 60$ to $950 \mathrm{~km} \mathrm{~s}^{-1}$ with a median value of $344.6 \mathrm{~km} \mathrm{~s}^{-1}$. The median virial radius and the median virial mass are $0.78 \mathrm{Mpc}^{-1}$ and $4.6 \times 10^{13} M_{\odot} h^{-1}$, respectively.

With the non-parametric $1 / V_{\max }$ method, we computed the mass function of galaxy systems in the SSC region, and we 
found evidences of a possible bias in the number of low mass systems. This effect could be a consequence of the correlation between the redshift completeness and the galaxy density, present in the Proust et al. (2004) compilation. By integrating the derived mass function in the range $10^{13}$ to $10^{15} M_{\odot} h^{-1}$, we found that the total mass of the SSC is on the order of $4.8 \times 10^{15} M_{\odot} h^{-1}$. Taking the possible bias in the identification of low mass systems into account and also that we are missing the mass outside galaxy systems, the last value should be taken as a lower boundary. Using the mock catalogues free of the redshift incompleteness, we found a mass of $\sim 1.1 \times 10^{16} M_{\odot} h^{-1}$ in the same mass range as before. We estimated the fraction of galaxies/mass that, according to the numerical simulation, is outside galaxy systems. With this fraction to correct the SSC mass, we found a total mass of $\sim 1.6 \times 10^{16} M_{\odot} h^{-1}$. In order to compare with the mass computed in R2000 using a spherical collapse model, we disregarded group peculiar motions and summed up the group virial masses. After correcting for the mass outside systems, we obtained $3.8 \times 10^{15} M_{\odot} h^{-1}$ for the SSC in the central $8 \mathrm{Mpc} h^{-1}$ sphere, in agreement with their work.

Acknowledgements. The authors wish to thank the referee Dr. $\mathrm{S}$. Bardelli for useful suggestions. The simulations used in this paper were carried out by the Virgo Supercomputing Consortium using computers at Computing Center of the Max-Planck Society in Garching and at the Edinburgh Parallel Computing Centre. The data are publicly available at www.mpa-garching.mpg.de/NumCos. This work was partially supported by the Agencia Nacional de Promoción Científica y Técnica, Secretaría de Ciencia y Técnica (SeCyT), Consejo de Investigaciones Científicas y Técnicas de la República Argentina (CONICET), Agencia Córdoba Ciencia, FONDAP Center for Astrophysics and FONDECYT grant \# 1020840.

\section{References}

Bardelli, S., Zucca, E., Zamorani, G., Vettolani, G., \& Scaramella, R. 1998, MNRAS, 296, 599

Bardelli, S., Zucca, E., Zamorani, G., Moscardini, L., \& Scaramella, R. 2000, MNRAS, 312, 540

Bardelli, S., Zucca, E., \& Baldi, A. 2001, MNRAS, 320, 387

Huchra, J. P., \& Geller, M. J. 1982, ApJ, 257, 423

Diaferio, A., \& Geller, M. J. 1997, ApJ, 481, 633

Drinkwater, M. J., Proust, D., Parker, Q. A., Slezak, E., \& Quintana, H. 1999, PASA, 16, 113

Drinkwater, M. J., Parker, Q. A., Proust, D., Slezak, E., \& Quintana, H. 2004, PASA, 21, 89

Kaldare, R., Colles, M., Raychaudhury, S., \& Slezak, E. 1995, AJ, 110,463

Kull, A., \& Böhringer, H. 1999, A\&A, 341, 23

Madgwick, D., Lahav, O., Baldry, I. K., et al. (the 2dFGRS Team) 2002, MNRAS, 333, 133

Merchán, M. E., \& Zandivarez, A. 2002, MNRAS, 335, 216

Moore, B., Frenk, C. S., \& White, S. D. M. 1993, MNRAS, 261, 827

Nolthenius, R., \& White, S. 1987, MNRAS, 225, 505

Norberg, P., Baugh, C. M., Hawkins, E., et al. (the 2dFGRS Team) 2001, MNRAS, 328, 64

Proust, D., Quintana, H., \& Reisenegger, A. 2004, in preparation

Quintana, H., Ramirez, A., Melnick, J., Raychaudhury, S., \& Slezak, E. 1995, AJ, 110, 463

Quintana, H., Melnick, J., Proust, D., \& Infante, L. 1997, A\&AS, 125, 247

Quintana, H., Carrasco, E. R., \& Reisenegger, A. 2000, AJ, 120, 511

Reisenegger, A., Quintana, H., Carrasco, E. R., \& Maze, J. 2000, AJ, 120,523

Rauzy, S. 2001, MNRAS, 324, 51

Watson, F. G., Parker, Q. A., et al. 2000, Proc SPIE, 4008, 123

Yoshida, N., Sheth, R., \& Diaferio, A. 2001, MNRAS, 328, 669 


\section{Online Material}


Table 1. Properties of SSGC groups.

\begin{tabular}{|c|c|c|c|c|c|c|c|}
\hline $\begin{array}{l}\text { SSGC } \\
\text { number }\end{array}$ & $\begin{array}{l}\text { RA } \\
(2000)\end{array}$ & $\begin{array}{l}\text { Dec } \\
(2000)\end{array}$ & $\begin{array}{l}\left\langle V_{\mathrm{r}}\right\rangle \\
{\left[\mathrm{km} \mathrm{s}^{-1}\right]}\end{array}$ & $\begin{array}{r}\sigma_{\mathrm{r}} \\
{\left[\mathrm{km} \mathrm{s}^{-1}\right]}\end{array}$ & $\begin{array}{r}\text { Mass } \\
{\left[\times 10^{13} M_{\odot}\right]}\end{array}$ & \#mem. & $\begin{array}{r}R_{\mathrm{vir}} \\
{[\mathrm{Mpc}]}\end{array}$ \\
\hline 1 & 185.799 & -34.508 & 2799 & 179 & 3.19 & 10 & 1.42 \\
\hline 2 & 187.332 & -39.023 & 3277 & 170 & 3.19 & 14 & 1.56 \\
\hline 3 & 191.622 & -41.408 & 3322 & 732 & 76.24 & 30 & 2.03 \\
\hline 4 & 190.763 & -36.761 & 3293 & 97 & 0.18 & 4 & 0.28 \\
\hline 5 & 192.786 & -26.389 & 3169 & 85 & 0.39 & 5 & 0.78 \\
\hline 6 & 193.412 & -28.312 & 16223 & 389 & 1.51 & 6 & 0.14 \\
\hline 7 & 193.425 & -28.817 & 16566 & 169 & 0.03 & 4 & 0.01 \\
\hline 8 & 193.635 & -29.042 & 16395 & 540 & 9.20 & 36 & 0.45 \\
\hline 9 & 193.553 & -29.841 & 21147 & 338 & 0.19 & 5 & 0.02 \\
\hline 10 & 193.598 & -30.570 & 15383 & 148 & 0.14 & 5 & 0.09 \\
\hline 11 & 193.678 & -28.985 & 22582 & 366 & 15.94 & 4 & 1.70 \\
\hline 12 & 194.133 & -30.292 & 16413 & 526 & 16.70 & 58 & 0.86 \\
\hline 13 & 193.831 & -30.514 & 16122 & 98 & 0.01 & 4 & 0.01 \\
\hline 14 & 194.106 & -31.310 & 16541 & 524 & 12.86 & 23 & 0.67 \\
\hline 15 & 194.342 & -32.792 & 22808 & 332 & 10.06 & 8 & 1.30 \\
\hline 16 & 194.280 & -30.380 & 15436 & 147 & 0.11 & 4 & 0.07 \\
\hline 17 & 194.446 & -29.878 & 17133 & 336 & 8.07 & 7 & 1.02 \\
\hline 18 & 194.360 & -29.036 & 18112 & 453 & 1.41 & 5 & 0.09 \\
\hline 19 & 194.513 & -28.502 & 20241 & 437 & 2.65 & 14 & 0.19 \\
\hline 20 & 194.529 & -28.215 & 22733 & 635 & 18.63 & 4 & 0.66 \\
\hline 21 & 194.764 & -28.129 & 14677 & 57 & 0.12 & 4 & 0.54 \\
\hline 22 & 195.290 & -35.498 & 5064 & 371 & 9.64 & 4 & 0.99 \\
\hline 23 & 195.118 & -37.339 & 4369 & 491 & 19.10 & 4 & 1.13 \\
\hline 24 & 194.909 & -30.493 & 17281 & 146 & 1.23 & 4 & 0.82 \\
\hline 25 & 195.505 & -32.259 & 4786 & 241 & 1.92 & 7 & 0.47 \\
\hline 26 & 195.200 & -31.361 & 3548 & 144 & 0.04 & 5 & 0.02 \\
\hline 27 & 195.357 & -33.981 & 14704 & 126 & 1.59 & 4 & 1.42 \\
\hline 28 & 196.075 & -30.154 & 3391 & 300 & 2.69 & 26 & 0.42 \\
\hline 29 & 195.525 & -32.424 & 9567 & 280 & 0.41 & 4 & 0.07 \\
\hline 30 & 195.408 & -30.925 & 5193 & 132 & 0.06 & 6 & 0.05 \\
\hline 31 & 195.581 & -32.858 & 5024 & 189 & 0.30 & 6 & 0.12 \\
\hline 32 & 195.563 & -32.895 & 9323 & 211 & 1.10 & 4 & 0.35 \\
\hline 33 & 195.901 & -31.472 & 16468 & 298 & 4.32 & 5 & 0.69 \\
\hline 34 & 195.888 & -34.011 & 24113 & 226 & 0.77 & 7 & 0.21 \\
\hline 35 & 196.179 & -30.640 & 15472 & 250 & 3.89 & 6 & 0.89 \\
\hline 36 & 196.179 & -32.209 & 9480 & 155 & 0.31 & 6 & 0.18 \\
\hline 37 & 196.539 & -37.610 & 14912 & 726 & 39.37 & 22 & 1.06 \\
\hline 38 & 196.465 & -31.497 & 16191 & 202 & 0.26 & 6 & 0.09 \\
\hline 39 & 196.499 & -34.264 & 25883 & 173 & 0.29 & 5 & 0.13 \\
\hline 40 & 197.069 & -28.227 & 2217 & 87 & 0.04 & 7 & 0.08 \\
\hline 41 & 196.541 & -29.145 & 15021 & 278 & 1.34 & 8 & 0.24 \\
\hline 42 & 196.576 & -29.571 & 20468 & 500 & 13.11 & 6 & 0.75 \\
\hline 43 & 196.844 & -31.758 & 15580 & 388 & 7.97 & 5 & 0.75 \\
\hline 44 & 197.134 & -32.077 & 9751 & 101 & 0.58 & 4 & 0.82 \\
\hline 45 & 197.161 & -34.430 & 15192 & 270 & 2.56 & 5 & 0.50 \\
\hline 46 & 197.799 & -34.715 & 29405 & 945 & 151.08 & 4 & 2.42 \\
\hline 47 & 197.892 & -34.343 & 27159 & 724 & 57.04 & 4 & 1.55 \\
\hline 48 & 199.394 & -43.473 & 3508 & 438 & 13.25 & 8 & 0.98 \\
\hline 49 & 198.483 & -30.207 & 32134 & 358 & 8.85 & 5 & 0.98 \\
\hline 50 & 198.761 & -33.616 & 14331 & 263 & 4.64 & 4 & 0.96 \\
\hline 51 & 198.858 & -32.668 & 14597 & 350 & 5.75 & 9 & 0.67 \\
\hline 52 & 199.728 & -25.120 & 1683 & 524 & 26.33 & 6 & 1.37 \\
\hline 53 & 199.658 & -37.252 & 15055 & 523 & 17.72 & 9 & 0.92 \\
\hline
\end{tabular}


Table 1. continued.

\begin{tabular}{|c|c|c|c|c|c|c|c|}
\hline $\begin{array}{l}\text { SSGC } \\
\text { number }\end{array}$ & $\begin{array}{l}\text { RA } \\
(2000)\end{array}$ & $\begin{array}{l}\text { Dec } \\
(2000)\end{array}$ & $\begin{array}{l}\left\langle V_{\mathrm{r}}\right\rangle \\
{\left[\mathrm{km} \mathrm{s}^{-1}\right]}\end{array}$ & $\begin{array}{r}\sigma_{\mathrm{r}} \\
{\left[\mathrm{km} \mathrm{s}^{-1}\right]}\end{array}$ & $\begin{array}{r}\text { Mass } \\
{\left[\times 10^{13} M_{\odot}\right]}\end{array}$ & \#mem. & $\begin{array}{r}R_{\mathrm{vir}} \\
{[\mathrm{Mpc}]}\end{array}$ \\
\hline 54 & 200.031 & -27.576 & 1889 & 270 & 0.60 & 7 & 0.11 \\
\hline 55 & 199.757 & -34.566 & 7974 & 380 & 3.48 & 5 & 0.34 \\
\hline 56 & 199.886 & -33.428 & 14928 & 323 & 5.82 & 9 & 0.80 \\
\hline 57 & 200.205 & -33.926 & 14251 & 495 & 13.62 & 6 & 0.79 \\
\hline 58 & 200.203 & -35.123 & 15033 & 229 & 4.34 & 4 & 1.17 \\
\hline 59 & 200.299 & -31.108 & 14713 & 447 & 9.87 & 6 & 0.70 \\
\hline 60 & 200.466 & -30.165 & 14627 & 305 & 4.67 & 4 & 0.71 \\
\hline 61 & 200.387 & -29.022 & 14005 & 129 & 0.08 & 5 & 0.06 \\
\hline 62 & 200.459 & -31.435 & 14798 & 382 & 9.49 & 4 & 0.93 \\
\hline 63 & 200.529 & -29.498 & 10004 & 133 & 0.58 & 4 & 0.47 \\
\hline 64 & 200.730 & -34.911 & 8025 & 223 & 1.22 & 5 & 0.35 \\
\hline 65 & 200.938 & -31.731 & 14454 & 491 & 16.59 & 37 & 0.98 \\
\hline 66 & 200.699 & -33.402 & 14231 & 419 & 6.21 & 7 & 0.50 \\
\hline 67 & 201.256 & -30.196 & 4250 & 220 & 0.67 & 15 & 0.19 \\
\hline 68 & 201.322 & -33.725 & 7785 & 134 & 0.83 & 4 & 0.65 \\
\hline 69 & 201.790 & -27.299 & 13690 & 787 & 47.09 & 43 & 1.08 \\
\hline 70 & 201.472 & -31.843 & 14370 & 395 & 1.40 & 6 & 0.12 \\
\hline 71 & 201.527 & -27.136 & 12160 & 225 & 2.52 & 4 & 0.71 \\
\hline 72 & 201.563 & -28.827 & 14179 & 212 & 1.47 & 4 & 0.46 \\
\hline 73 & 201.937 & -31.567 & 14094 & 910 & 67.17 & 91 & 1.16 \\
\hline 74 & 201.837 & -27.359 & 10515 & 378 & 10.02 & 16 & 1.00 \\
\hline 75 & 201.919 & -30.635 & 14514 & 377 & 1.33 & 6 & 0.13 \\
\hline 76 & 201.891 & -29.313 & 4156 & 338 & 3.27 & 4 & 0.40 \\
\hline 77 & 202.187 & -27.929 & 10299 & 327 & 4.63 & 8 & 0.61 \\
\hline 78 & 202.009 & -31.035 & 13560 & 512 & 15.70 & 5 & 0.85 \\
\hline 79 & 202.063 & -34.006 & 14727 & 369 & 6.35 & 5 & 0.66 \\
\hline 80 & 202.255 & -33.507 & 14197 & 344 & 5.86 & 4 & 0.70 \\
\hline 81 & 202.335 & -31.521 & 14913 & 576 & 30.25 & 26 & 1.30 \\
\hline 82 & 202.607 & -31.946 & 14325 & 763 & 47.19 & 25 & 1.16 \\
\hline 83 & 202.807 & -31.720 & 13819 & 682 & 37.26 & 26 & 1.14 \\
\hline 84 & 202.508 & -29.531 & 14298 & 300 & 1.96 & 9 & 0.31 \\
\hline 85 & 202.454 & -31.220 & 16187 & 368 & 7.38 & 5 & 0.77 \\
\hline 86 & 202.554 & -28.224 & 10039 & 308 & 1.40 & 7 & 0.21 \\
\hline 87 & 202.726 & -30.496 & 14818 & 354 & 8.88 & 5 & 1.01 \\
\hline 88 & 203.169 & -33.123 & 14553 & 606 & 34.15 & 33 & 1.33 \\
\hline 89 & 202.909 & -32.994 & 3575 & 113 & 0.06 & 6 & 0.07 \\
\hline 90 & 203.416 & -31.590 & 14547 & 650 & 26.52 & 14 & 0.89 \\
\hline 91 & 203.661 & -35.160 & 14895 & 297 & 1.19 & 7 & 0.19 \\
\hline 92 & 203.635 & -33.037 & 14991 & 165 & 1.59 & 4 & 0.83 \\
\hline 93 & 203.901 & -33.895 & 3646 & 282 & 3.93 & 10 & 0.70 \\
\hline 94 & 203.913 & -31.869 & 14356 & 145 & 1.19 & 8 & 0.80 \\
\hline 95 & 203.874 & -31.447 & 14435 & 377 & 3.28 & 5 & 0.33 \\
\hline 96 & 204.840 & -35.617 & 15398 & 503 & 17.06 & 23 & 0.96 \\
\hline 97 & 205.178 & -34.052 & 14807 & 373 & 13.84 & 11 & 1.42 \\
\hline 98 & 204.981 & -29.747 & 23296 & 618 & 13.21 & 4 & 0.49 \\
\hline 99 & 205.537 & -30.708 & 4587 & 140 & 1.04 & 9 & 0.75 \\
\hline 100 & 205.591 & -30.040 & 14460 & 359 & 7.91 & 4 & 0.87 \\
\hline 101 & 205.900 & -29.906 & 23380 & 219 & 3.98 & 4 & 1.18 \\
\hline 102 & 206.459 & -29.950 & 4450 & 73 & 0.24 & 8 & 0.65 \\
\hline 103 & 206.472 & -32.168 & 14946 & 183 & 0.17 & 4 & 0.07 \\
\hline 104 & 206.917 & -32.880 & 11996 & 589 & 31.28 & 55 & 1.29 \\
\hline 105 & 206.645 & -31.632 & 11400 & 407 & 2.31 & 8 & 0.19 \\
\hline 106 & 207.251 & -30.563 & 4539 & 503 & 12.01 & 37 & 0.67 \\
\hline 107 & 206.926 & -32.660 & 10424 & 163 & 1.80 & 7 & 0.96 \\
\hline
\end{tabular}


Table 1. continued.

\begin{tabular}{llllrrrr}
\hline \hline $\begin{array}{l}\text { SSGC } \\
\text { number }\end{array}$ & RA & $\begin{array}{l}\text { Dec } \\
(2000)\end{array}$ & $\begin{array}{l}\left\langle V_{\mathrm{r}}\right\rangle \\
{\left[\mathrm{km} \mathrm{s}^{-1}\right]}\end{array}$ & $\begin{array}{r}\sigma_{\mathrm{r}} \\
{\left[\mathrm{km} \mathrm{s}^{-1}\right]}\end{array}$ & $\begin{array}{r}\text { Mass } \\
{\left[\times 10^{13} M_{\odot}\right]}\end{array}$ & \#mem. & $\begin{array}{r}R_{\text {vir }} \\
{[\mathrm{Mpc}]}\end{array}$ \\
\hline 108 & 207.162 & -28.938 & 14773 & 123 & 0.31 & 9 & 0.29 \\
109 & 207.691 & -48.476 & 3022 & 263 & 3.14 & 5 & 0.65 \\
110 & 207.389 & -32.243 & 11265 & 545 & 22.52 & 4 & 1.08 \\
111 & 207.484 & -30.207 & 22294 & 496 & 27.91 & 8 & 1.62 \\
112 & 207.955 & -30.635 & 4438 & 368 & 6.76 & 10 & 0.71 \\
113 & 207.590 & -32.853 & 11043 & 528 & 21.00 & 5 & 1.07 \\
114 & 208.152 & -28.642 & 4895 & 312 & 4.58 & 12 & 0.67 \\
115 & 208.189 & -32.865 & 11204 & 210 & 2.45 & 5 & 0.79 \\
116 & 208.404 & -27.876 & 15069 & 413 & 20.64 & 14 & 1.73 \\
117 & 208.362 & -30.340 & 22259 & 271 & 8.33 & 8 & 1.61 \\
118 & 208.643 & -26.578 & 5703 & 232 & 4.71 & 5 & 1.25 \\
119 & 210.625 & -34.035 & 4220 & 419 & 17.65 & 39 & 1.43 \\
120 & 209.597 & -32.562 & 4402 & 161 & 1.47 & 6 & 0.80 \\
121 & 210.572 & -33.149 & 11012 & 334 & 9.34 & 5 & 1.19 \\
122 & 211.985 & -27.024 & 6773 & 386 & 7.15 & 13 & 0.68 \\
\hline
\end{tabular}


Table 2. Groups or clusters of galaxies previously identified close to SSGC groups.

\begin{tabular}{|c|c|c|c|c|}
\hline $\begin{array}{l}\text { SSGC } \\
\text { number }\end{array}$ & System name & $\begin{array}{l}\text { Type of } \\
\text { system }\end{array}$ & $\begin{array}{l}\text { Mean } V_{\mathrm{r}} \\
{\left[\mathrm{km} \mathrm{s}^{-1}\right]}\end{array}$ & $\begin{array}{r}\Delta \theta \\
\text { [arc. min.] }\end{array}$ \\
\hline SSGC 3 & LGG 301 & GGroup & & 10.6 \\
\hline \multirow{2}{*}{ SSGC 5} & USGC S187 & GGroup & 3337 & 5.3 \\
\hline & ABELL S0714 & GClstr & 3258 & 6.0 \\
\hline SSGC 7 & ABELL 3528 & GClstr & 15829 & 14.6 \\
\hline \multirow[t]{2}{*}{ SSGC 8} & ABELL 3528N & GClstr & 15889 & 3.3 \\
\hline & AM 1252-285 & GGroup & $\ldots$ & 8.4 \\
\hline SSGC 12 & ABELL 3530 & GClstr & 16099 & 12.4 \\
\hline SSGC 15 & ABELL 3531 & GClstr & 22454 & 8.2 \\
\hline SSGC 16 & ABELL 3532 & GClstr & 16609 & 2.6 \\
\hline SSGC 17 & LGG 317 & GGroup & $\ldots$ & 4.0 \\
\hline SSGC 19 & ABELL 3535 & GClstr & 19546 & 3.3 \\
\hline SSGC 20 & ABELL S0717 & GClstr & $\ldots$ & 0.3 \\
\hline SSGC 25 & AM $1258-320$ & GGroup & $\ldots$ & 11.1 \\
\hline SSGC 28 & NGC 4936 GROUP & GGroup & $\ldots$ & 8.3 \\
\hline SSGC 29 & ABELL 3537 & GClstr & 9593 & 13.4 \\
\hline SSGC 30 & AM 1258-303 & GGroup & $\ldots$ & 3.9 \\
\hline SSGC 35 & LGG 326 & GGroup & $\ldots$ & 19.2 \\
\hline \multirow[t]{2}{*}{ SSGC 37} & ABELL S0721 & GClstr & 14690 & 1.7 \\
\hline & VV 671 & GGroup & $\ldots$ & 1.9 \\
\hline SSGC 40 & NGC 4965 GROUP & GGroup & $\ldots$ & 9.6 \\
\hline SSGC 45 & ABELL 3542 & GClstr & 15739 & 8.2 \\
\hline SSGC 47 & ABELL 3545 & GClstr & 28270 & 15.8 \\
\hline SSGC 49 & ABELL S0725 & GClstr & $\ldots$ & 3.1 \\
\hline \multirow[t]{2}{*}{ SSGC 50} & ABELL S0726 & GClstr & 17700 & 2.7 \\
\hline & SCL 128 & GClstr & $\ldots$ & 23.6 \\
\hline SSGC 51 & SCL 129 & GClstr & $\ldots$ & 19.2 \\
\hline \multirow[t]{2}{*}{ SSGC 53} & AM 1315-365 & GGroup & $\ldots$ & 2.0 \\
\hline & ABELL 3553 & GClstr & 14600 & 8.5 \\
\hline SSGC 54 & ABELL S0728 & GClstr & $\ldots$ & 18.6 \\
\hline \multirow[t]{2}{*}{ SSGC 56} & ABELL 3554 & GClstr & 14090 & 3.1 \\
\hline & AM 1316-330 NED02 & GGroup & $\ldots$ & 4.6 \\
\hline SSGC 61 & ABELL 3555 & GClstr & 14630 & 10.5 \\
\hline SSGC 62 & [BPR98] B0109 & GGroup & $\ldots$ & 11.7 \\
\hline SSGC 64 & AM 1320-343 & GGroup & $\ldots$ & 2.5 \\
\hline \multirow[t]{2}{*}{ SSGC 65} & [BPR98] T075 & GGroup & 15075 & 4.9 \\
\hline & ABELL 3556 & GClstr & 14360 & 6.2 \\
\hline SSGC 66 & AM 1320-330 & GGroup & $\ldots$ & 0.9 \\
\hline \multirow[t]{2}{*}{ SSGC 67} & CL 1322-30 & GClstr & 4222 & 6.6 \\
\hline & AM 1322-300 & GGroup & $\ldots$ & 7.8 \\
\hline SSGC 69 & AM 1324-265 & GClstr & $\ldots$ & 9.3 \\
\hline SSGC 70 & [BPR98] T260 & GGroup & 1472 & 14.6 \\
\hline SSGC 71 & ABELL 1736 NED02 & GClstr & 13577 & 9.4 \\
\hline \multirow[t]{2}{*}{ SSGC 73} & [BPR98] T300 & GGroup & 14952 & 3.4 \\
\hline & ABELL 3558 & GClstr & 14390 & 5.0 \\
\hline SSGC 74 & ABELL 1736 NED01 & GClstr & 10443 & 9.4 \\
\hline SSGC 78 & [BPR98] B1014 & GGroup & $\ldots$ & 3.4 \\
\hline \multirow[t]{2}{*}{ SSGC 81} & SC1327-312 & GCstr & 14481 & 7.7 \\
\hline & [BPR98] T413 & GGroup & 15099 & 8.0 \\
\hline SSGC 82 & [BPR98] T496 & GGroup & 14673 & 8.8 \\
\hline \multirow[t]{2}{*}{ SSGC 83} & [BPR98] T520 & GGroup & 13446 & 3.3 \\
\hline & SC1329-313 & GGroup & 14450 & 7.3 \\
\hline \multirow[t]{2}{*}{ SSGC 84} & HCG 065 & GGroup & 14240 & 1.6 \\
\hline & ABELL 3559 & GClstr & 13820 & 2.1 \\
\hline
\end{tabular}


Table 2. continued.

\begin{tabular}{lllrr}
\hline \hline SSGC & System name & $\begin{array}{l}\text { Type of } \\
\text { number }\end{array}$ & $\begin{array}{r}\text { Mean } V_{\mathrm{r}} \\
{\left[\mathrm{km} \mathrm{s}^{-1}\right]}\end{array}$ & $\begin{array}{r}\Delta \theta \\
\text { [arc. min.] }\end{array}$ \\
\hline SSGC 86 & ABELL S0736 & GClstr & $\ldots$ & 15.0 \\
SSGC 88 & RX J1332.2-3303 & GClstr & 14090 & 7.7 \\
& ABELL 3560 & GClstr & 14660 & 11.9 \\
SSGC 90 & [BPR98] T561 & GGroup & 14501 & 1.8 \\
& ABELL 3562 & GClstr & 14690 & 5.3 \\
SSGC 91 & AM 1331-311 NED01 & GGroup & $\ldots$ & 7.3 \\
SSGC 93 & ABELL 3565 & GClstr & 15140 & 5.0 \\
SSGC 94 & [BPR98] T599 & GClstr & 3687 & 14.0 \\
SSGC 95 & [BPR98] T598 & GGroup & 14426 & 2.5 \\
SSGC 96 & ABELL 3566 & GClstr & 14901 & 13.9 \\
SSGC 98 & AM 1337-292 & GGroup & $\ldots .4$ \\
& SC 1340-294 & GClstr & 23229 & 1.2 \\
SSGC 100 & SC 1342-302 & GClstr & 14515 & 1.5 \\
SSGC 104 & ABELL 3571 & GClstr & 11722 & 18.3 \\
SSGC 106 & LGG 359 & GGroup & $\ldots$ & 2.6 \\
SSGC 109 & AM 1346-483 & GGroup & $\ldots$ & 19.5 \\
SSGC 112 & AM 1349-300 & GGroup & $\ldots$ & 23.2 \\
SSGC 113 & AM 1346-324 & GGroup & $\ldots$ & 16.0 \\
SSGC 114 & KLEMOLA 28 & GGroup & $\ldots$ & 11.5 \\
SSGC 115 & ABELL 3575 & GClstr & 11302 & 2.2 \\
SSGC 116 & ABELL 3577 & GClstr & 14810 & 9.8 \\
SSGC 117 & AM 1349-300 & GGroup & $\ldots$ & \\
SSGC 119 & ABELL S0753 & GClstr & 4197 & 6.3 \\
SSGC 122 & ABELL 3581 & GClstr & 6895 & \\
\hline
\end{tabular}

